\title{
Enhanced nose-to-brain delivery of tranilast using liquid crystal formulations
}

Gerard Lee See ${ }^{a, c}$, Florencio Arce, Jr. ${ }^{a, c}$, Sabrina Dahlizar ${ }^{a, d}$, Akie Okada ${ }^{a}$, Muhammad Fikri Bin Mohd Fadli ${ }^{e}$, Ichiro Hijikuro $f$, Shoko Itakura ${ }^{a, b}$, Masanori Katakura ${ }^{a, b}$, Hiroaki Todo ${ }^{a, b}$, $5 \quad$ Kenji Sugibayashi ${ }^{a, b^{*}}$

${ }^{a}$ Graduate School of Pharmaceutical Sciences, Josai University, Saitama, Japan.

${ }^{b}$ School of Pharmacy and Pharmaceutical Sciences, Josai University, Saitama, Japan.

${ }^{c}$ Department of Pharmacy, School of Health Care Professions, University of San Carlos, Cebu,

10 the Philippines.

${ }^{d}$ Department of Pharmacy, Faculty of Health Science, Syarif Hidayatullah State Islamic University Jakarta, Banten, Indonesia.

${ }^{e}$ School of Pharmacy, Management and Science University, Selangor, Malaysia.

${ }^{f}$ Farnex Inc., Tokyo Institute of Technology, Yokohama Venture Plaza, Nagatsuta-cho, Midori-

$15 k u$, Yokohama, Japan

*Corresponding author at: School of Pharmacy and Pharmaceutical Sciences, Josai

University, Japan.

E-mail address: sugib@josai.ac.jp (K. Sugibayashi).

Highlights

- Intranasal delivery of tranilast was enhanced by MGE and GMO liquid crystals

- Biodistribution of administered LC formulations was visualized by micro-computed tomography tandem in vivo imaging systems

- MGE and GMO formed liquid crystal structures with formulation characteristics supporting brain uptake of lipophilic drugs 


\section{ABSTRACT}

Intranasal administration is poised as a competent method in delivering drugs to the brain, because the nasal route has a direct link with the central nervous system bypassing the formidable blood-brain barrier. $\mathrm{C}_{17}$-monoglycerol ester (MGE) and glyceryl monooleate (GMO) as liquid crystal (LC)-forming lipids possess desirable formulation characteristics as drug carriers for intranasally administered drugs. This study investigated the effect of LC formulations on the pharmacokinetics of tranilast (TL), a lipophilic model drug, and its distribution in the therapeutic target regions of the brain in rats. The anatomical biodistribution of LC formulations was monitored using micro-computed tomography tandem in vivo imaging systems. MGE and GMO effectively formed LC with suitable particle size, zeta potential, and viscosity supporting the delivery of TL to the brain. MGE and GMO LC formulations enhanced brain uptake by 10 - to 12 -fold and 2- to 2.4 - fold, respectively, compared with TL solution. The olfactory bulb had the highest TL concentration and fluorescent signals among all the brain regions, indicating a direct nose-to-brain delivery pathway of LC formulations. LC-forming lipids, MGE and GMO, are potential biomaterials in formulations intended for intranasal administration.

Keywords: intranasal drug delivery; liquid crystals; micro-computed tomography; in vivo imaging; C-17 monoglycerol ester; tranilast 


\section{INTRODUCTION}

Delivering drugs to the central nervous system (CNS) in the management of acute and chronic neurodegenerative disorders remains an unmet clinical need owing to the blood-brain barrier, wherein numerous tight junctions and efflux transporters inhibit molecules from entering the brain, hence limiting therapies for many CNS diseases [1]. Oral routes of administration, the use of implanted medical devices, and intrathecal and intracerebroventricular injections have been used as methods to deliver drugs to the brain. Although these methods are used in clinical applications, they are associated with drawbacks such as invasiveness, low central bioavailability, and requiring clinical supervision during administration $[2,3]$.

Nasal drug administration is poised as a competent alternative to the currently available techniques, because drugs delivered by the intranasal route can be delivered directly to the brain and bypassing the blood-brain barrier. The direct anatomical link between the nasal cavity and the CNS makes it feasible to deliver many drugs via this route. Several studies have established that drugs reach the CNS from the nasal cavity by direct transport along olfactory and trigeminal pathways [4-7]. In addition, the nasal route of administration usually results in higher bioavailability, thereby requiring lower doses to be administered due to rapid absorption without enzymatic degradation and, in turn, reduced toxicity. The nasal route is noninvasive, can be self-administered, and may be better received by patients resulting in improved compliance [8-10].

Liquid crystal (LC) systems have received significant attention as carriers for the controlled release of drugs because their unique microstructures have a range of desirable characteristics [11]. LC phases in formulations are thermodynamically stable, biocompatible, and contain extremely large surface areas with the ability to incorporate numerous drugs independently of their solubilities [12,13]. Moreover, LC systems can spontaneously form 
either lamellar, cubic, or hexagonal crystalline mesophases by adding biocompatible amphiphilic lipids, such as glyceryl monooleate (GMO) and $\mathrm{C}_{17}-$ monoglycerol ester (MGE), to an aqueous environment. LC systems formed by GMO and water have been extensively investigated about their ability to sustain the release of a wide range of drugs. The use of GMO in LC systems has been applied in various administration routes, comprising oral, transdermal, and ophthalmic delivery, but there have been no studies with regards to its usefulness for noseto-brain delivery $[11,14]$.

Tranilast (TL), a lipophilic molecule, was selected as a model drug for this study because its clinical uses have extended beyond bronchial asthma, atopic dermatitis and allergic conjunctivitis [15]. Several studies have demonstrated that TL suppresses inducible nitric oxide synthase and activity by interferon- $\gamma$. Hence, the potential for TL in treating various CNS conditions associated with nitric oxide production including multiple sclerosis, cerebral ischemia, and Alzheimer's disease is underway [16,17]. Additionally, the purported applications of TL in the treatment of Alzheimer's disease has been appropriately patented [18]. Therefore, the critical value of enhanced delivery of TL to the brain must be explored.

In this study, we developed LC formulations of TL using a novel LC-forming lipid,

100 GMO and MGE, intended to be administered intranasally. Previously, we reported that an MGE-based LC had an ideal size range and zeta potential suitable as a candidate drug carrier for intranasally administered drugs. MGE was selected as an LC-forming lipid for its stability and better drug absorption enhancement compared with other LC-forming lipids [19]. Our study aimed to establish the effect of LC formulations on the distribution of TL to specific brain regions after intranasal administration in rats. TL concentration has been quantified in therapeutic target regions (i.e., hippocampus, cortex) in the brain. Lastly, the biodistribution of LC formulations from the nose to the brain was verified using micro-computed tomography $(\mu \mathrm{CT})$ tandem in vivo imaging systems. 


\section{MATERIALS AND METHODS}

110

\section{Materials and animals}

TL was purchased from Tokyo Chemical Industry Co. Ltd. (Tokyo, Japan). Pluronic ${ }^{\circledR}$ F-127 was purchased from Sigma-Aldrich (St. Louis, MO., U.S.A.). C17-monoglycerol ester (MGE) and glyceryl monooleate (GMO) were provided by Farnex Inc. (Yokohama, Japan). A

115 near-infrared fluorescent cyanine dye, 1,1'-dioctadecyltetramethylindotricarbocyanine iodide (DiR) was purchased from Thermo Fisher Scientific (Waltham, MA., U.S.A.). Ethanol (99.5\%) and acetonitrile (99.8\%) were purchased from Fujifilm Wako Chemical Corp. (Osaka, Japan).

Male Sprague Dawley rats (7 weeks of age, body weight $230 \pm 10$ g) were obtained from Sankyo Laboratory Service Corporation, Inc. (Tokyo, Japan). Animals were housed in a 120 controlled environment $\left(25^{\circ} \mathrm{C}, 12 \mathrm{~h}\right.$ light-dark cycle $)$ with free access to food and water. Rats were anesthetized with three types of anesthesia $(0.375 \mathrm{mg} / \mathrm{kg}$ medetomidine, $2.5 \mathrm{mg} / \mathrm{kg}$ butorphanol, and $2 \mathrm{mg} / \mathrm{kg}$ midazolam) by intraperitoneal route (i.p.) prior to experiments.

The animal experiment protocol was approved by the Animal Care and Use Committee of Josai University with the approval number JU18003.

\section{Preparation and characterization of LC formulations}

The compositions of LC formulations prepared in this study are presented in Table 1. The selection of components and proportions of the formulations were based on our previous work on MGE LC formulations with slight modification [19]. GMO was melted at $80{ }^{\circ} \mathrm{C}$ before use. The components were combined and the resulting mixture was sonicated using a probe sonicator (VCX-750, Sonics \& Materials, Inc., Newton, CT, U.S.A.) at an amplitude of $20 \%$ for 5 mins. TL in solution (TLC), which served as the control, was prepared by dissolving TL with a sufficient amount of phosphate-buffered saline (PBS) $\mathrm{pH} 7.4$. 


\section{Measurement of vesicle size, polydispersity index, and zeta potential}

The vesicle size, polydispersity index (PDI), and zeta potential of the prepared LC formulations ( $\mathrm{TL}_{\mathrm{M}}, \mathrm{TL}_{\mathrm{ME}}, \mathrm{TL}_{\mathrm{G}}, \mathrm{TL}_{\mathrm{GE}}$ ) were determined using a dynamic light scattering NanoZS ZEN3600 Zetasizer (Malvern Instruments Ltd., Worcestershire, U.K.). Formulations were diluted with purified water and shaken using a vortex mixer prior to measurement.

140 Measurement of vesicle size, PDI, and zeta potential was done in triplicate at room temperature.

\section{Measurement of viscosity}

The viscosity of LC formulations was determined using a viscometer (Toki Sangyo Co., Ltd, Tokyo, Japan) with a sensitivity range of $0.3-10,000 \mathrm{mPa} \cdot \mathrm{s}$ and with an accuracy of $1 \%$ 145 relative error.

\section{Small Angle X-ray scattering (SAXS) analysis}

The prepared LC formulations were subjected to small angle X-ray scattering (SAXS) measurement using a Nano-viewer (Rigaku, Akishima Tokyo, Japan) with a Pilatus 100K/RL

150 2D detector. The X-ray source was $\mathrm{Cu} \mathrm{K} \alpha$ radiation with a wavelength of $1.54 \AA$ and operating at $45 \mathrm{kV}$ and $110 \mathrm{~mA}$. The sample-to-detector distance was set at $375 \mathrm{~nm}$. The individual sample was placed in a vacuum - resistant glass capillary cell and exposed at $25^{\circ} \mathrm{C}$ for $1 \mathrm{~h}$. The SAXS pattern obtained was plotted against the scattering vector length, $q=(4 \pi / \lambda) \sin (\theta / 2)$, where $\theta$ is the scattering angle. The scattering intensity was normalized using the decayed 155 direct beam intensity.

\section{Entrapment efficiency}


In the measurement of entrapment efficiency, LC formulations were centrifuged at $15,000 \mathrm{rpm}$ for $15 \mathrm{~min}$ at $4^{\circ} \mathrm{C}$. An aliquot of the resulting supernatant was withdrawn and

160 diluted with ten-fold acetonitrile and quantified using liquid chromatography-tandem mass spectroscopy (LC-MS/MS). Entrapment efficiency was calculated based on the equation below:

$$
\% E E=\frac{T L_{\text {total }}-T L_{\text {free }}}{T L_{\text {total }}} * 100
$$

where $\mathrm{EE}, \mathrm{TL}_{\text {total }}, \mathrm{TL}_{\text {free }}$ are entrapment efficiency, total $\mathrm{TL}$ concentration in formulation, and concentration of TL in the supernatant, respectively.

\section{In vitro drug release from the $\mathrm{LC}$ formulations}

In vitro drug release of the formulations was evaluated using a dialysis membrane (MW cut-off; 2,000 - 14,000 Da, Sanko Junyaku Co., Tokyo, Japan) previously hydrated and set on a side-by-side diffusion cell (effective diffusion area: $0.95 \mathrm{~cm}^{2}$ ). PBS was filled into the receiver chamber. The receiver chamber was thermostatically maintained at $37^{\circ} \mathrm{C}$. LC formulations $(3.0 \mathrm{~mL})$ were applied to the donor cell to commence the release experiment. Sampling was performed by withdrawal of an aliquot $(500 \mu \mathrm{L})$ at pre-determined time points. An equal volume of PBS was added to the receiver chamber to keep the volume constant 175 throughout the experiment.

\section{Tissue distribution studies of TL formulations}

Rats were randomly divided into five groups. $\mathrm{TL}_{\mathrm{M}}, \mathrm{TL}_{\mathrm{ME}}, \mathrm{TL}_{\mathrm{G}}, \mathrm{TL}_{\mathrm{GE}}$, and $\mathrm{TL}_{\mathrm{C}}(0.5$ $\mathrm{mg} / \mathrm{kg}$ ) were intranasally administered to the first, second, third, fourth and fifth groups,

180 respectively. Ten microliters of either $\mathrm{LC}$ formulation or TLC was administered dropwise using a micropipette (Eppendorf Reference $2^{\circledR}$, Eppendorf AG, Hamburg, Germany) inserted (0.5 
$\mathrm{cm}$ ) into each nostril with an interval of $60 \mathrm{~s}$. Rats were kept in supine position throughout the experiment. A separate group of rats was allocated for intravenous administration, the tranilast solution $(0.5 \mathrm{mg} / \mathrm{kg})$ was administered through the tail vein.

At predetermined time points $(0.17,0.5,1,2,4,8 \mathrm{~h})$, approximately $200 \mu \mathrm{L}$ of blood was withdrawn from the jugular vein and directly transferred into heparinized tubes and immediately centrifuged $\left(15,000 \mathrm{rpm} ; 10 \mathrm{~min} ; 4^{\circ} \mathrm{C}\right)$ to obtain plasma. After this, the same volume of normal saline solution was infused via the tail vein to circumvent alterations in the volume of distribution. After blood collection at 2, 4, and $8 \mathrm{~h}$, rats were deeply anesthetized with three types of anesthesia i.p. as abovementioned and transcardially perfused with cold PBS to avoid blood from contaminating the brain. The whole brain was isolated and dissected on ice into specific regions (olfactory bulb, cortex, brainstem, cerebellum, midbrain, hippocampus). The spinal cord was also collected. The collected brain samples were weighed then reduced in size using scissors, and $0.5 \mathrm{~mL}$ of acetonitrile was added prior to homogenization at $12,000 \mathrm{rpm}$ and $4^{\circ} \mathrm{C}$ for 5 min using a homogenizer (Polytron PT $1200 \mathrm{E}$, Kinematica AG, Littaue-Lucerne, Switzerland). The brain homogenates were then centrifuged at $15,000 \mathrm{rpm}$ and $4^{\circ} \mathrm{C}$ for $5 \mathrm{~min}$. The plasma and brain samples were kept at $-30^{\circ} \mathrm{C}$ until analysis.

The peak plasma concentration $\left(C_{\max }\right)$ was determined directly from plasma or brain 200 concentration-time curves. The area under the plasma or brain concentration-time curve $\left(A U C_{0-}\right.$ 8) was calculated using trapezoidal rule [20-22]. Drug targeting efficiency percentage (DTE) and nose-to-brain direct transport percentage $(\% D T P)$ were calculated according to previous methods described elsewhere $[23,24]$.

$205 \% D T E=\frac{\left(\frac{A U C_{\text {brain }}}{A U C_{\text {blood }}}\right) \text { intranasal }}{\left(\frac{A U C_{\text {brain }}}{A U C_{\text {blood }}}\right) \text { intravenous }} \cdot 100 \quad$ Eq. 1 
$\% D T P=\frac{B_{i n}-B_{x}}{B_{\text {in }}} \cdot 100$

Eq. 2

$B_{x}=\left(\frac{B_{i v}}{P_{i v}}\right) \cdot P_{\text {in }} \quad$ Eq. 3

where $B_{x}$ is the brain AUC fraction contributed by the systemic circulation through the BloodBrain Barrier following intranasal administration, $B_{i n}$ is the brain AUC over time following intranasal administration, $P_{i v}$ is the blood AUC over time following intravenous administration, and $P_{i n}$ is the blood AUC over time following intranasal administration [24].

\section{Sample preparation and quantification of TL using LC-MS/MS}

Supernatant $(50 \mu \mathrm{L})$ from plasma or brain homogenates or samples from the in vitro release experiment were mixed with an equal amount of acetonitrile and centrifuged at $4{ }^{\circ} \mathrm{C}$ for 5 min. The resulting supernatant $(10 \mu \mathrm{L})$ was injected into an LC/MS/MS system for the quantification of TL. The LC/MS/MS systems consisted of a system controller (CBM-20A; Shimadzu Corporation, Kyoto, Japan), pump (LC-20AD; Shimadzu), auto-sampler (SIL20ACHT; Shimadzu), column oven (CTO-20A; Shimadzu), detector (4000QTRAP; AB Sciex;

220 Tokyo, Japan), and analysis software (Analyst ${ }^{\circledR}$ version 1.4.2; Shimadzu). The column (Shodex ODP2HPG-2A $2.0 \mathrm{~mm}$ x $10 \mathrm{~mm}$, Showa Denko Inc; Tokyo, Japan) was kept at $40^{\circ} \mathrm{C}$. The mobile phase used was acetonitrile: $0.05 \%$ formic acid containing $5 \mathrm{mM}$ ammonium acetate (80:20). The flow rate was maintained at $0.2 \mathrm{~mL} / \mathrm{min}$. Mass spectrometric quantification was carried out in the multiple reaction monitoring (MRM) mode, monitoring transition ions of $\mathrm{m} / \mathrm{z}$ 328.0 to $\mathrm{m} / \mathrm{z}, 191.2$ with collision energy of $36 \mathrm{eV}$. Calibration was carried out with six different concentrations within a range of $1-1000 \mathrm{ng} / \mathrm{mL}$ with a correlation coefficient of 0.9999 .

In vivo biodistribution studies of $\mathrm{LC}$ formulations using combined in vivo imaging system and $\mu \mathrm{CT}$. 

dye when incorporated into lipids. DiR-labeled MGE (DiR $\left.\mathrm{D}_{\mathrm{M}}, \mathrm{DiR}_{\mathrm{ME}}\right)$ and GMO $\left(\mathrm{DiR}_{\mathrm{G}}, \mathrm{DiR}_{\mathrm{GE}}\right)$ LC formulations were prepared using the quantities described in Table 1 without TL. DiR solution $\left(\mathrm{DiR}_{\mathrm{S}}\right)(0.2 \mathrm{mM})$ was prepared by diluting DiR ethanol solution $(10 \mathrm{mM})$ with a sufficient amount of water.

Sprague Dawley rats were anesthetized and divided into five groups; $\mathrm{DiR}_{\mathrm{M}}, \mathrm{DiR}_{\mathrm{ME}}$, $\mathrm{DiR}_{\mathrm{G}}, \mathrm{DiR}_{\mathrm{GE}}, \mathrm{DiR}$. Before administration, rats were subjected to X-ray $\mu \mathrm{CT}$ imaging using CosmoScan GX II system (Rigaku Corp., Akishima, Tokyo, Japan). Cranial bone images of rats were obtained using the following parameters: $90 \mathrm{kV}$ of $\mathrm{X}$-ray tube voltage, $88 \mu \mathrm{A}$ of $\mathrm{X}$ ray tube current. Then, $\mathrm{DiR}_{\mathrm{M}}, \mathrm{DiR}_{\mathrm{ME}}, \mathrm{DiR}_{\mathrm{G}}, \mathrm{DiR}_{\mathrm{GE}}, \mathrm{DiR}_{\mathrm{S}}$ were administrated intranasally to

240 the assigned rats identically to the abovementioned procedure in the pharmacokinetics study. After administration, in vivo fluorescent images of rats were obtained at 0, 15, 30, 60 and 120 min using the IVIS ${ }^{\circledR}$ Spectrum in vivo imaging system (PerkinElmer ${ }^{\circledR}$ Ltd., Waltham, MA, USA). To visualize the fluorescence signals of formulations in $3 \mathrm{D}$ anatomical context, fluorescence signals were co-registered with the anatomical $\mu \mathrm{CT}$ images using Multi-Modality Module Software (PerkinElmer ${ }^{\circledR}$ Ltd., Waltham, MA, USA).

At 120 min post-intranasal administration, rats were sacrificed and the whole brain was carefully excised to avoid contamination. Ex vivo fluorescent imaging of the whole brain was conducted with the IVIS ${ }^{\circledR}$ Spectrum in vivo imaging system. The following conditions were used for image acquisition: $745 \mathrm{~nm}$ excitation and $800 \mathrm{~nm}$ emission filters, auto exposure time and F/Stop $=2$. The fluorescence images were analyzed by Living Image ${ }^{\circledR} 4.7 .2$ software (PerkinElmer ${ }^{\circledR}$ Ltd., Waltham, MA, USA). All experiments were conducted in three trials.

\section{Statistical analysis}


Statistical analyses were performed using Student's $t$-test $(p<0.05)$. All data were expressed as the mean with standard error (mean $\pm \mathrm{SE}$ ).

\section{RESULTS}

\section{Characterization of $\mathrm{LC}$ formulations}

$T L_{M}$ and $T L_{M E}$ formulations were opaque white dispersion without visible aggregates, whereas $\mathrm{TL}_{\mathrm{G}}$ and $\mathrm{TL}_{\mathrm{GE}}$ formulations were viscous, pale yellow dispersions without visible aggregates, and all formulations were stable throughout the experiment. $\mathrm{TL}_{\mathrm{C}}$ was a clear colorless solution. Table 2 shows the mean vesicle size, PDI, zeta potential, viscosity, and entrapment efficiency of the prepared formulations. PDI values of MGE and GMO-based formulations indicated homogenous particle size distribution. Negative zeta potential is good for fine dispersion of the formulation. Addition of ethanol ( $T L_{M}$ to $T L_{M E}, T_{G}$ to $T L_{G E}$ ) markedly increased the viscosity of the formulations. Entrapment efficiency of MGE- and GMO-based formulations exhibited good incorporation of TL in the LC structure. The phase structure of TL formulations was evaluated using SAXS and X-ray diffraction profiles showed

270 typical peaks reflection patterns at nearly $1, \sqrt{3}$, and $\sqrt{4}$ for $\mathrm{TL}_{\mathrm{M}}$ suggesting an inverse hexagonal type of LC (Fig. 1). TLME revealed peaks reflection patterns at $\sqrt{2}, \sqrt{3}, \sqrt{4}$, $\sqrt{6}, \sqrt{8}, \sqrt{9}$ suggesting a bicontinuous cubic $(\mathrm{Pn} 3 \mathrm{~m})$ type of $\mathrm{LC}$. TL $\mathrm{G}$ showed peaks reflection patterns at $\sqrt{2}, \sqrt{4}, \sqrt{6}$ indicating a bicontinuous cubic $(\operatorname{Im} 3 \mathrm{~m})$ LC, whereas TLGE had unverified reflection patterns. DiR-labeled LC formulations had identical peak reflection 275 patterns with their respective TL LC formulations (data not shown). Particle size, PDI, and zeta potential of DiR-labeled LC formulations had indistinguishable variances with their respective TL LC formulations (data not shown). 


\section{Drug release profile of $\mathrm{LC}$ formulations}

Figure 2 shows the release profile of TL from the LC formulations. The cumulative amount of TL released over $8 \mathrm{~h}$ from $\mathrm{TL}_{\mathrm{M}}, \mathrm{TL}_{\mathrm{ME}}, \mathrm{TL}_{\mathrm{G}}$ and $\mathrm{TL}_{\mathrm{GE}}$ were $389.75 \pm 23.06 \mu \mathrm{g} / \mathrm{cm}^{2}$, $547.70 \pm 12.33 \mu \mathrm{g} / \mathrm{cm}^{2}, 185.24 \pm 9.39 \mu \mathrm{g} / \mathrm{cm}^{2}, 268.56 \pm 22.41 \mu \mathrm{g} / \mathrm{cm}^{2}$, respectively. This corresponds to a 2-fold higher release rates in MGE formulations when compared with GMO formulations. For both MGE and GMO formulations, addition of ethanol increased the release rate of TL.

\section{TL distribution in plasma and whole brain}

Brain uptake of TL following intranasal administration on Sprague Dawley rats was observed for a duration of $8 \mathrm{~h}$. Figure $3 \mathrm{~A}$ and $\mathrm{B}$ show the TL concentration in plasma and the

290 whole brain, respectively, following intranasal administration. Table 3 presents the pharmacokinetic parameters. $\mathrm{TL}_{\mathrm{M}}$ and $\mathrm{TL}_{\mathrm{ME}}$ formulations exhibited a sustained $\mathrm{TL}$ concentration profile in the brain (Fig. 3B). TLME formulations had $A U C_{(0-8)}$ values of 94.5 and $114 \mathrm{ng} \cdot \mathrm{h} / \mathrm{mL}$ in the whole brain, which corresponded to 10 - to 12 -fold higher TL concentration compared with TLC. TLME showed the highest $C_{\max }$ in the brain. Mean residence

295 time of TL from LC formulations in the brain ranged from 4 to $6 \mathrm{~h}$ corresponding to 2- to 3fold higher residence time in the brain than in plasma.

\section{TL distribution in brain regions}

Figure 4 shows the TL distribution in different regions of the brain following intranasal

300 administration. All brain regions showed uptake of TL at $2 \mathrm{~h}$, the first collection point. Formulations containing MGE and GMO exhibited increasing TL concentrations in different brain regions over time, but $\mathrm{TL}_{\mathrm{C}}$ showed a decreasing trend. An apparent selective distribution of TL from LC formulations to brain regions was seen from 4 to $8 \mathrm{~h}$. Among the brain regions, 
the olfactory bulb showed the highest TL concentration of $76.3 \mathrm{ng} / \mathrm{g}$ and $18.2 \mathrm{ng} / \mathrm{g}$ at $2 \mathrm{~h}$

following the intranasal administration of $\mathrm{TL}_{\mathrm{M}}$ and $\mathrm{TL}_{\mathrm{ME}}$, respectively. $\mathrm{TL}_{\mathrm{M}}$ showed a decreasing TL concentration in the olfactory bulb over time whereas TL concentrations in the hippocampus increased over time. In addition, higher TL concentrations at $8 \mathrm{~h}$ were observed in the hippocampus and spinal cord for MGE LC formulations. Calculated \%DTE and \%DTP are presented in Table 3.

310

\section{In vivo biodistribution studies of LC formulations}

Figure 5 shows in vivo fluorescent images of DiR-labeled LC formulations combined with $\mu \mathrm{CT}$. The combination in vivo imaging system and $\mu \mathrm{CT}$ imaging allowed the visualization and monitoring of the distribution of formulations in a 3D anatomical context. A fluorescent dye, DiR, was incorporated to lipid-based LC formulations to understand the disposition of administered formulations from the nasal cavity to the brain. The movement and location of fluorescence signals in $\mathrm{DiR}_{\mathrm{M}}$ and $\mathrm{DiR}_{\mathrm{ME}} \mathrm{LC}$ formulations were traced from the administration site to the adjacent regions of the olfactory region, and finally in the olfactory bulb. $\mathrm{DiR}_{\mathrm{G}}$ and $\mathrm{DiR}_{\mathrm{GE}} \mathrm{LC}$ formulation had strong fluorescence that remained stationary in the nasal cavity (see Fig. 5B). DiR solution ( $\left.\mathrm{DiR}_{\mathrm{S}}\right)$ exhibited rapid elimination from the nasal cavity and generated low fluorescence signals. Moreover, slice images of rat cranium in the lateral direction (Fig. 5B) revealed the localization of the fluorescence. Ex vivo images of the whole brain had strong fluorescence in the olfactory bulb and brain stem from $\mathrm{DiR}_{M}$ but detectable fluorescence in the olfactory bulb was only found from $\mathrm{DiR}_{\mathrm{ME}} \mathrm{LC}$ formulation at 120 mins post-administration (Fig. 5C).

\section{DISCUSSION}


The usefulness of MGE- and GMO-based LC as nanocarriers in enhancing the delivery of TL into the brain and its specific regions after intranasal administration was investigated in this study. TL concentrations in specific brain regions were determined instead of the whole brain alone in an attempt to understand the distribution pathway of TL from LC formulations. In addition, MGE- and GMO-based formulations containing ethanol were also prepared to clarify the influence of ethanol on the properties of LC systems and its impact on nose-to-brain delivery, because the mixture of water and ethanol is a common solvent in dissolving lipophilic drugs, such as TL, in pharmaceuticals.

MGE and GMO lipids with TL ( $\mathrm{TL}_{\mathrm{M}}$ and $\mathrm{TL}_{\mathrm{G}}$ ) formed an $\mathrm{H}_{2}$ inverted hexagonal phase LC and Im3M body-centered cubic phase LC, respectively, whereas the addition of ethanol to MGE (TLME) formed a Pn3m bicontinuous double-diamond cubic structure, suggesting that ethanol possibly modulates the transitions in LC structures (see Fig. 1). An aqueous dispersion of TL consisting of nanoparticles with an internal reversed hexagonal structure, hexosomes, or reversed internal bicontinuous cubic phase, cubosomes, were successfully prepared in this study. Hexosomes and cubosomes function as versatile platforms for drug delivery owing to their capacity to incorporate drugs independently of their solubilities and their large surface areas to interact with biological membranes [25].

MGE (TLM, TLME) and GMO ( $\left.\mathrm{TL}_{\mathrm{G}}, \mathrm{TL}_{\mathrm{GE}}\right) \mathrm{LC}$ formulations were characterized in terms of particle size, PDI, zeta potential, viscosity, and entrapment efficiency (Table 2). The particle size of prepared LC formulations conformed with the ideal nanocarrier size of 150 $400 \mathrm{~nm}$, which can efficiently deliver drug payload into the brain $[5,9,10,26]$. PDI values indicated a homogenous particle size distribution in the formulations. The zeta potential of the prepared LC formulations had strong negative values, suggesting predominance of repulsive forces at the surface of the LC, which resisted aggregation of particles and yielded better stability of the formulation $[27,28]$. Viscosity values of the LC formulations were significantly 
different from the control. Formulations containing GMO and ethanol had higher viscosities. MGE and GMO formulations had higher entrapment efficiency by 1 to 1.5 -fold compared with their ethanol-containing formulations, which might support their function as a carrier of TL from nose to brain, and the ability of the LC formulation to release TL was confirmed in our in vitro release studies. Bicontinuous $\mathrm{LC}$ structures $\left(\mathrm{TL}_{\mathrm{ME}}, \mathrm{TL}_{\mathrm{G}}\right)$ had higher drug release rates compared with the inverse hexagonal LC $\left(\mathrm{TL}_{\mathrm{M}}\right)$ structure, which was possibly related to the differences in the internal structures of the LC involved. In addition, the entrapment efficiency and in vitro release data indicated that sufficient concentrations of TL were detected in the brain despite lower volumes of the formulation (10 $\mathrm{L}$ /nostril) administered intranasally than previous reports $(25-60 \mu \mathrm{L} /$ nostril $)[20,29]$.

In vivo studies on the nose-to-brain delivery of TL were implemented to understand how $\mathrm{LC}$ formulations affect the distribution of TL in the brain. TL in solution (TLC) exhibited $365 A U C$ values of $189 \mathrm{ng} \cdot \mathrm{h} / \mathrm{mL}$ in the plasma and $9.55 \mathrm{ng} \cdot \mathrm{h} / \mathrm{mL}$ in the brain, which corresponded to a 20-fold higher plasma concentration, indicating that TL rapidly entered the systemic circulation after intranasal administration but had poor localization in the brain (Table 3 ). On the other hand, MGE and GMO LC formulations rendered TL with higher transport efficiency in the brain which corresponds to 10 - to 12 -fold and 2- to 2.4- fold higher brain uptake,

370 respectively, when compared with $\mathrm{TL}_{\mathrm{C}}$ (Table 3). TL concentrations in the brain were higher compared to plasma for $\mathrm{TL}_{\mathrm{M}}$, while otherwise was observed for TLME. $\mathrm{TL}_{\mathrm{G}}$ and $\mathrm{TL}_{\mathrm{GE}}$ formulations yielded low concentrations of TL in the brain and plasma, which was attributable to their high viscosities. GMO-based LC formulations had 2- to 3-fold higher viscosity than MGE-based LC formulations. In addition, viscosity may have contributed to the delayed time 375 to reach peak concentration in the brain, as observed in GMO-based LC formulations. Of note, MGE-based LC formulations showed faster systemic and brain transport than GMO-based formulations wherein differences in the morphology of the LC may explain this finding. 
Furthermore, LC formulations exhibited longer residence time in the brain than $\mathrm{TL}_{\mathrm{C}}$, signifying a slow absorption rate and subsequently a slow elimination process, enabling TL accumulation in the brain. In a previous study, oral administration of TL was reported to have a low brain tissue-to-plasma concentration ratio $(K p)$ of 0.38 [30]. In our study, a lower $K p$ value was observed in $\mathrm{TL}_{\mathrm{C}}(0.13)$ after intranasal administration. However, $K p$ values of intranasally administered LC formulations were 0.46 - 3.4 (Table 3), corresponding to 1.2- to 9-fold higher TL concentrations in the brain than the oral route. The intranasal route of administration proved to be superior over oral routes of administration in the delivery of TL to the brain.

Findings from the biodistribution studies revealed that GMO-based formulations ( $\mathrm{DiR}_{\mathrm{G}}$ and $\mathrm{DiR}_{\mathrm{GE}}$ ) remained in contact with the nasal cavity over a period of $2 \mathrm{~h}$ with sustained fluorescence intensity, indicating that the drug remained at the application site (Fig. 5A). On the other hand, the $\mathrm{DiR}_{M}$ and $\mathrm{DiR}_{\mathrm{ME}}$ formulations were observed to diffuse gradually from the nasal cavity into the olfactory region, suggesting the controlled release of MGE LC formulations. DiR $R_{S}$ transited into the pharynx rapidly indicating fast elimination of the solution from the application site (Fig. 5A, B). Co-registered fluorescence with X-ray and $\mu$ CT images of the cranium confirmed the localization of DiR in the rostral sections of the brain from MGEbased LC formulations. Ex vivo brain images of rats administered DiR-labeled MGE LC formulations had higher fluorescence than their ethanol counterparts (Fig. 5C). This affirmed our data on TL tissue distribution for $\mathrm{TL}_{\mathrm{M}}$ where higher brain targeting and a lower systemic absorption were observed compared with TLME. In addition, the differences in the LC structures possibly influenced the localization and extent of biodistribution of LC formulations where $\mathrm{DiR}_{\mathrm{M}}$ (inverse hexagonal) and $\mathrm{DiR}_{\mathrm{ME}}$ (bicontinuous cubic, $\operatorname{Im} 3 \mathrm{~m}$ ) showed higher brain penetration.

Formulation design plays an important role in targeted drug delivery into specific regions of the brain (i.e. hippocampus and cortex in Alzheimer's disease; midbrain in 
Parkinson's disease) in mitigating neurodegenerative diseases. TL concentrations from MGEbased LC formulations in different brain regions were significantly higher compared with the

405 GMO-based LC formulations and control at $8 \mathrm{~h}$ (Fig. 3B). TL was detected in brain regions of therapeutic interest, particularly the midbrain, cortex, and hippocampus (Fig. 4). Among the regions of the brain, the olfactory bulb had the highest TL concentration from MGE-based LC formulations (Fig. 4). In addition, ex vivo brain images of $\mathrm{DiR}_{M}$ and $\mathrm{DiR}_{\mathrm{ME}}$ showed strong fluorescence localized in the olfactory bulb and brain stem (Fig. 5C). This evidence confirmed the direct pathway to support nose-to-brain delivery of drugs by LC formulations.

Transport of drugs into the brain from the olfactory region of the nasal cavity is supported by two known pathways, the olfactory and trigeminal pathways. The olfactory ensheathing cells and neural fibroblasts in the olfactory region are structures continuous with the meninges covering the brain, and thus capable of transporting drugs directly into the brain

415 following intranasal administration [31]. TL concentration in whole brain for $\mathrm{TL}_{\mathrm{M}}$ formulation peaked at $2 \mathrm{~h}$, and dissection of brain regions revealed that TL concentrations in olfactory bulb peaked at $2 \mathrm{~h}$ as well (Fig. 3). In addition, DiR $\mathrm{M}_{\mathrm{M}}$ exhibited strong fluorescence in the olfactory bulb, which was confirmed in the biodistribution studies and ex vivo brain images at $2 \mathrm{~h}$ (Fig. 4). This suggested that a direct nose-to-brain delivery of TL exists via the olfactory pathway for the $\mathrm{TL}_{\mathrm{M}}$ formulation. $\mathrm{TL}_{\mathrm{M}}$ is a less viscous formulation that tended to flow freely and diffused sufficiently to cover a large surface area of the olfactory region. On the other hand, the high concentrations of TL found in plasma (Fig. 2A) and the brain stem (Fig. 3) presented a different distribution pathway for TL from $\mathrm{TL}_{\mathrm{ME}}$, with the respiratory epithelium possibly influencing its transport. Moreover, the high viscosity of the TLME formulation (Table 2) may facilitate prolonged contact with the lateral walls of the nasal cavity of the anterior respiratory epithelium instead of the olfactory region, which is located at the far superior aspect of the nasal cavity. The large and highly vascularized surface area of the respiratory epithelium, a 
known absorption site of drugs, could have been the gateway for the systemic absorption of TL from TLME. In the case of TLME formulation, TL was solubilized in ethanol effectively and the presence of MGE acting as a membrane permeation enhancer may have facilitated the absorption of TL through the respiratory epithelium. Moreover, trigeminal nerves (maxillary branch), originating from the brain stem, innervate the respiratory epithelium and may facilitate the transport of TL into the brain via the trigeminal pathway. It must be noted that the olfactory pathway delivers drug to the rostral (front) sections of the brain, whereas the trigeminal pathway delivers to both rostral and caudal (back) sections of the brain, making it difficult to account for which pathway facilitated drug transport into the rostral section [26]. Both MGEbased LC formulations showed high brain uptake. However, this phenomenon can be explained by two different pathways of absorption. The $K p$ value of $T L_{M}$ was $2.8,2.5$, and 3.4 at 2, 4 and $8 \mathrm{~h}$, respectively, whereas TLME had $K p$ values of $0.2,0.3$, and 0.7 , respectively. The 440 calculated $K p$ value of $\mathrm{TL}_{\mathrm{ME}}$ at $2 \mathrm{~h}$ was closely related to the $K p$ value obtained for $\mathrm{TL}_{\mathrm{C}}(0.13)$, indicating that the systemic circulation contributed to TL brain uptake in the first few hours, and a direct nose-to-brain route contributed to TL in the brain at a later time, as shown by the increasing $K p$ values over time. TLM had high $K p$ values maintained over time, with a predominantly direct nose-to-brain migration proposed for $\mathrm{TL}_{\mathrm{M}}$, as indicated by its higher TL concentration in brain than in plasma. Moreover, \%DTP supports this finding wherein $73.9 \%$ of TL from TLM at $8 \mathrm{~h}$ were attributed to a direct nose-to-brain pathway (Table 3). TL concentration in the brain for $\mathrm{TL}_{\mathrm{ME}}$ had contributions from direct nose-to brain migration as well as TL penetrating the brain via systemic circulation, as indicated by the higher plasma concentration obtained for $\mathrm{TL}_{\mathrm{ME}}$ (Fig.3A). Further investigations to clarify the distribution pathway of LC formulations in delivering drugs to the brain are needed.

Our previous works on MGE-based liquid crystals injectable formulations in in vivo experiments in rats demonstrated no severe toxicities (no weight loss, no respiratory failure 
and no inflammation at the administration site) while its antiadhesion formulation in a rat peritoneal adhesion model was found to be well tolerated with both studies spanning weeks of observation $[32,33]$. Isoprenoid-type compounds are metabolized by the liver and excreted by the kidney and intestinal tract. It is potentially predictable that MGE follows this particular metabolic pathway [33]. On the other hand, GMO is a nontoxic, biodegradable, and biocompatible material and is listed in the FDA's Inactive Ingredients Guide [34]. However, further studies are needed to be performed for better understanding of local (i.e., nasal ciliotoxicity studies), systemic toxicity and biocompatibility of intranasally administered liquid crystal formulations.

Formulation characteristics of MGE- and GMO-based LC formulations administered intranasally supported the delivery of drugs into the brain. This is the first study to demonstrate the usefulness of LC formulations in enhancing TL delivery into the brain following intranasal administration. In addition, this is a pioneering work utilizing the combined methods of an in vivo imaging system and $\mu \mathrm{CT}$ in visualizing the biodistribution of LC formulations to identify the anatomical localization of drugs. Although this is a putative evidence on the LC-mediated delivery of drugs into the brain by intranasal administration, further investigations focusing on optimizing the effects of LC-based drug carriers, understanding formulation mechanisms, and the dose needed to exhibit pharmacologic actions in brain disease models are recommended.

\section{CONCLUSION}

MGE and GMO effectively formed LCs with suitable formulation characteristics supporting the brain uptake of lipophilic drugs. Intranasal administration of LC formulations enhanced the delivery of TL into the brain and distribution to brain regions. The olfactory bulb consistently had the highest TL concentration and fluorescence signals among all the brain regions, indicating a direct nose-to-brain delivery pathway for the LC formulations. GMO- 
based LC formulations demonstrated the ability to sustain contact with the nasal cavity. MGEbased LC formulations can diffuse deep within the olfactory region and exhibit higher drug transport into the brain. LC-forming lipids, MGE and GMO, are potential biomaterials in formulations intended for intranasal administration. Biodistribution properties of intranasally administered LC formulations from the nasal cavity into the rostral section of the brain have been confirmed and visualized in real-time using an in vivo imaging system combined with $\mu \mathrm{CT}$.

\section{REFERENCES}

1. Gänger, S., Schindowski, K. 2018. Tailoring formulations for intranasal nose-to-brain delivery: a review on architecture, physicochemical characteristics and mucociliary clearance of the nasal olfactory mucosa. Pharmaceutics, 10:116, 1- 28.

2. Davanzo, J.R., Rizk, E. 2015. Baclofen overdose from possible intrinsic malfunction of SynchroMed II intrathecal pump : two case reports. J. Neurosurg. Pediatr., 16, 232 $-234$.

3. Awaad, Y., Rizk, T., Siddiqui, I., Roosen, N., Mcintosh, K., Waines, G.M. 2012. Complications of intrathecal baclofen pump : prevention and cure. ISRN Neurol, 2012, $1-6$.

4. Shingaki, T., Inoue, D., Furubayashi, T., Sakane, T., Katsumi, H., Yamamoto, A., Yamashita S. 2010. Transnasal delivery of methotrexate to brain tumors in rats: a new strategy for brain tumor chemotherapy. Mol. Pharm. 7, $1561-1568$.

500 5. Sonvico, F., Clementino, A., Buttini, F., Colombo, G., Pescina, S., Guterres S.S., Pohlmann, A.R., Nicoli, S. 2018. Surface-modified nanocarriers for nose-to-brain delivery: from bioadhesion to targeting. Pharmaceutics, 10:34, 1-34. 
6. Krishnan, J.K.S., Arun, P., Chembukave, B., Appu, A.P., Vijayakumar, N., Moffett, J.R., Puthillathu, N., Namboodiri, A.M.A. 2017. Effect of administration method, animal weight and age on the intranasal delivery of drugs to the brain. $\mathrm{J}$ Neurosci. Methods, 286, $16-21$.

7. Erdó, F., Bors, L.A., Farkas, D., Bajza, A., Sveinbjörn, G. 2018. Evaluation of intranasal delivery route of drug administration for brain targeting. Brain Res. Bull., $143,155-170$.

8. Espinoza, L.C., Silva-Abreu, M., Clares, B., Rodríguez-Lagunas, M.J., Halbaut, L., Cañas, M.A., Calpena, A.C. 2019. Formulation strategies to improve nose-to-brain delivery of donepezil. Pharmaceutics 11:64, 1- 16.

9. Warnken, Z.N., Smyth, H.D.C., Watts, A.B., Weitman, S., Kuhn, J.G., Williams, R.O. 2016. Formulation and device design to increase nose to brain drug delivery. J Drug Deliv Sci Technol. 35, $213-222$.

10. Katare, Y.H., Piazza, J.E., Bhandari, J., Daya, R.P., Akilan, K., Simpson, M.J., Hoare, T., Mishra, R.K. 2017. Intranasal delivery of antipsychotic drugs. Schizophrenia Res. $184,2-13$.

11. Souza, C., Watanabe, E., Borgheti-Cardoso, L.N., Fantini, M.C.D.A., Lara, M.G. 2014. Mucoadhesive system formed by liquid crystals for buccal administration of poly(hexamethylene biguanide) hydrochloride. J. Pharm. Sci. 103, 12, 3914 - 3923.

12. Chang, D.P., Barauskas, J., Dabkowska, A.P., Wadsäter, M., Tiberg, F., Nylander, T. 2015. Non-lamellar lipid liquid crystalline structures at interfaces. Adv. Colloid Interface Sci., 222, 135 - 147.

13. Garti, N., Libster, D., Aserin, A. 2014. Solubilization and delivery of drugs from GMObased lyotropic liquid crystals, in: Li, Q. (Ed.), Nanoscience with Liquid Crystals: from 
self-organized nanostructures to application. Springer International Publishing, Switzerland, pp. $355-409$.

14. Du, J.D., Liu, Q.T., Salentinig, S., Nguyen, T.H., Boyd, B.J. 2014. A novel approach to enhance the mucoadhesion of lipid drug nanocarriers for improved drug delivery to the buccal mucosa. Int. J. Pharm. 471, 358 - 365.

15. Darakhshan, S., Pour, A.B. 2015. Tranilast: a review of its therapeutic applications. Pharmacol Res., 91, $15-28$.

16. Platten, M., Eitel, K., Wischhusen, J., Dichgans, J., Weller, M. 2003. Involvement of protein kinase $c \delta$ and extracellular signal-regulated kinase- 2 in the suppression of microglial inducible nitric oxide synthase expression by $\mathrm{N}-[3,4$ dimethoxycinnamoyl]-anthranilic acid (tranilast). Biochem. Pharmacol., 66, 1236 1270.

17. Platten, M., Wick, W., Wischhusen, J., Weller, M. 2001. N-[3,4-dimethoxycinnamoyl]anthranilic acid (tranilast) suppresses microglial inducible nitric oxide synthase (iNOS) expression and activity induced by interferon- $\gamma($ INF- $\gamma$ ). Br. J. Pharmacol., 134, 1279 1284.

18. Schneider, A., Moraru, A., Krüger, C., Laage, R., Pitzer, C. Use of tranilast and derivatives thereof for the therapy of neurological conditions. United States Patent Application Publication (US $2011 / 0112187$ A1). https://patentimages.storage.googleapis.com/64/dd/8f/45ead2a80347ec/US201101121 87A1.pdf

19. Kadhum, W.R., Hada, T., Hijikuro, I., Todo, H., Sugibayashi, K. 2017. Development and optimization of orally and topically applied liquid crystal drug formulations. J Oleo Sci., 66, $939-950$. 
20. Liu, S., Yang, S., Ho, P.C. 2018. Intranasal administration of carbamazepine-loaded carboxymethyl chitosan nanoparticles for drug delivery to the brain. Asian J Pharm Sci., $13,72-81$.

21. Gao, M., Mei, D., Huo, Y., Mao, S. 2019. Effect of polysorbate 80 on the intranasal absorption and brain distribution of tetramethylpyrazine phosphate in rats. Drug Deliv Trans. Res., 9 , $311-318$.

22. See, G.L., Sagesaka, A., Todo, H., Wierzba, K., Sugibayashi, K. 2019. Pharmacokinetics and tissue distribution of pilocarpine after application to eyelid skin of rats. J. Pharm. Sci., 108, $2942-2948$.

23. Zhang, Q., Jiang, X., Jiang, W., Lu, W., Su, L., Shi, Z. 2004. Preparation of nimodipineloaded microemulsion for intranasal delivery and evaluation on the targeting efficiency to the brain. Int. J. Pharm. 275, $85-96$.

24. Kozlovskaya, L., Abou-Kaoud, M., Stepensky, D. 2014. Quantitative analysis of drug delivery to the brain via nasal route. J. Control Release. 189, 133 - 140.

25. Azmi, I.D.M., Moghimi, S.M., Yaghmur, A. 2015. Cubosomes and hexosomes as versatile platforms for drug delivery. Ther. Deliv. 6, 1347 - 1364.

26. Khan, A.R., Liu, M., Khan, M.W., Zhai, G. 2017. Progress in brain targeting drug delivery system by nasal route. J. Control Release, 268, 364 - 389.

27. Saraiva, C., Praça, C., Ferreira, R., Santos, T., Ferreira, L., Bernardino, L. 2016. Nanoparticle-mediated brain drug delivery: overcoming blood-brain barrier to treat neurodegenerative diseases. J. Control. Release, 235, 34 - 47.

28. Sharma, D., Sharma, R.K., Sharma, N., Gabrani, R., Sharma, S.K., Ali, J., Dang, S. 2015. Nose-to-brain delivery of PLGA-diazepam nanoparticles. AAPS Pharm Sci Tech., 16, $1108-1121$. 
29. Netsomboon, K., Partenhauser, A., Rohrer, J., Sündermann, N.E., Prüfert, F., Suchaoin, W., Laffleur, F., Bernkop-Schnürch, A. 2016. Preactivated thiomers for intranasal delivery of apomorphine: in vitro and in vivo evaluation. Eur. J. Pharm. Biopharm., 109, $35-42$.

30. Yang, W., Sabi-Mouka, E.M.B., Wang, L., Shu, C., Wang, Y., Ding, J., Ding, L. 2018. Determination of tranilast in bio-samples by LC-MS/MS: application to a pharmacokinetic and brain tissue distribution study in rats. J. Pharm. Biomed. Anal. $147,479-484$.

31. Crowe, T.P., Greenlee, H.W., Kanthasamy, A.G., Hsu, W.H. 2018. Mechanism of intranasal drug delivery to the brain. Life Sci, 195, $44-52$.

32. Okada, A., Todo, H., Hijikuro, I., Itakura, S., Sugibayashi, K. 2020. Controlled release of a model hydrophilic high molecular weight compound from injectable non-lamellar liquid crystal formulations containing different types of phospholipids. Int. J. Pharm., $577,118944$.

33. Murakami, T., Hijikuro, I., Yamashita, K., Tsunoda, S., Hirai, K., Suzuki, T., Sakai, Y., Tabata, Y. 2019. Antiadhesion effect of the C17 glycerin ester of isoprenoid-type lipid forming a nonlamellar liquid crystal. Acta Biomaterialia, 84, 257 - 267.

34. Chen, Y., Ma, P., Gui, S. 2014. Cubic and hexagonal liquid crystals as drug delivery systems. BioMed Res. Int., 815981. 


\section{FIGURES}
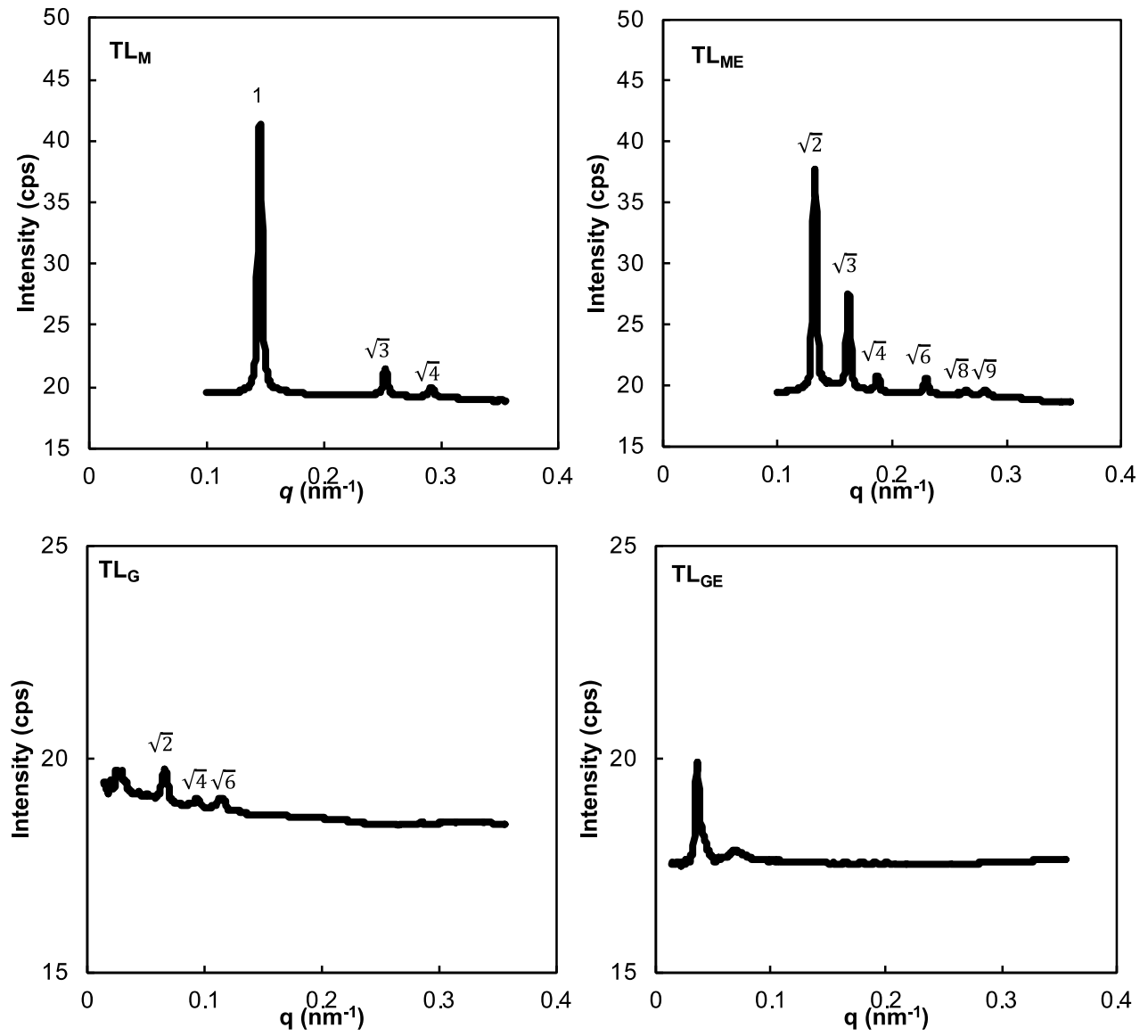

610

Figure 1. SAXS diffraction pattern of tranilast in MGE and GMO-based LC formulations. 


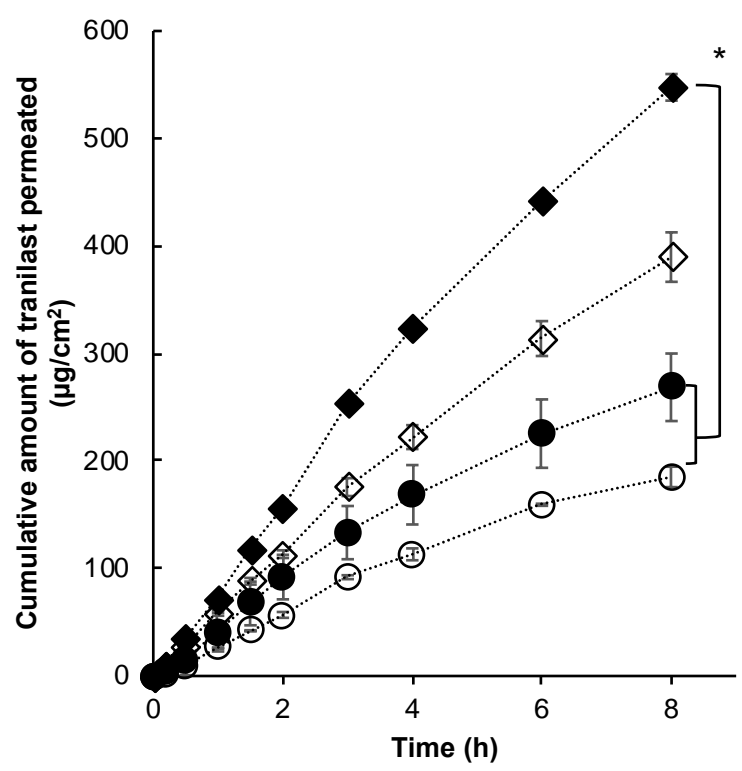

625

Figure 2. In vitro release profile of tranilast formulations. $\mathrm{TL}_{\mathrm{M}}(\diamond), \mathrm{TL}_{\mathrm{ME}}(\diamond), \mathrm{TL}_{\mathrm{G}}(\circ), \mathrm{TL}_{\mathrm{GE}}$ $(\bullet)$. Each value represents the mean \pm S.E. $(\mathrm{n}=3-4)$. Significant difference $\left({ }^{*} p \leq 0.05\right)$ between $\mathrm{TL}_{\mathrm{ME}}$ and $\mathrm{TL}_{\mathrm{G}}$ or TLGE.

630

A

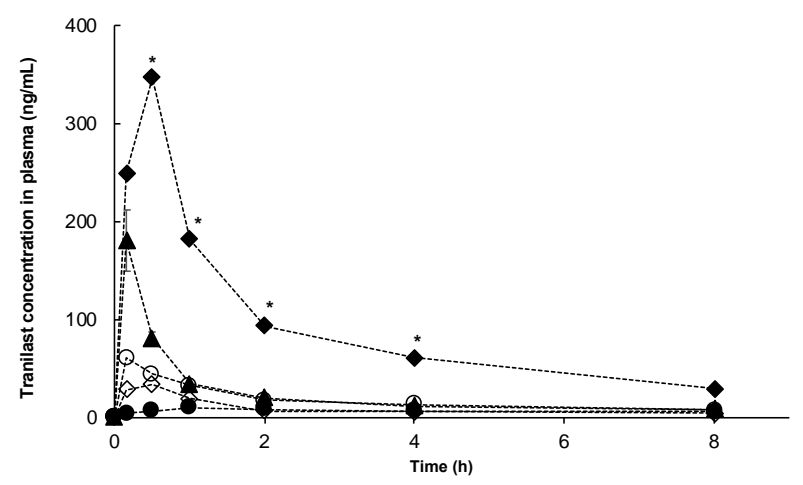

$\mathrm{B}$

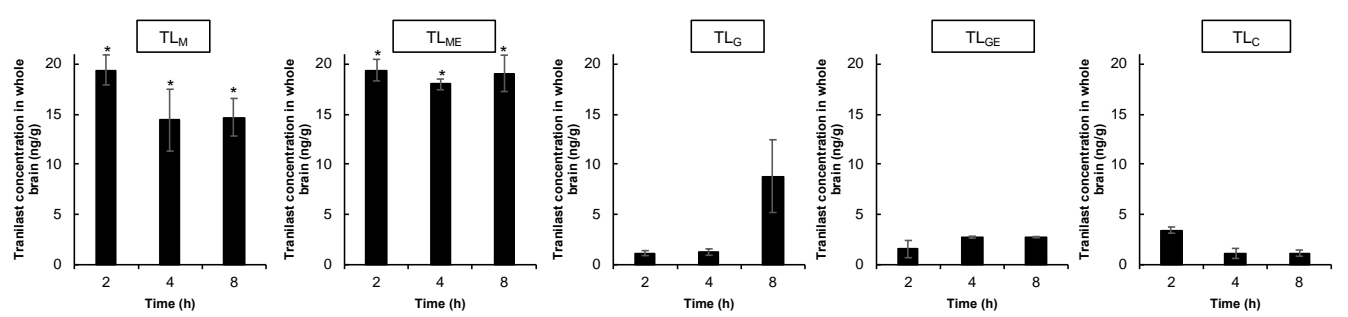

Figure 3. Concentration of tranilast in plasma (A) and whole brain (B). TLM $(\diamond), \operatorname{TL}_{\mathrm{ME}}(\bullet)$, $\mathrm{TL}_{\mathrm{G}}(\circ), \mathrm{TL}_{\mathrm{GE}}(\bullet), \mathrm{TL}_{\mathrm{C}}(\boldsymbol{\Delta})$. Each value represents the mean \pm S.E. $(\mathrm{n}=3-4)$. Significant difference $(* p \leq 0.05)$ between $\mathrm{TL}_{\mathrm{M}}$ and $\mathrm{TL}_{\mathrm{G}}$, TL $\mathrm{TL}_{\mathrm{GE}}$ or $\mathrm{TL}_{\mathrm{C}}$; $\mathrm{TL}_{\mathrm{ME}}$ and $\mathrm{TL}_{\mathrm{G}}, \mathrm{TL}_{\mathrm{GE}}$ or $\mathrm{TL}_{\mathrm{C}}$. 

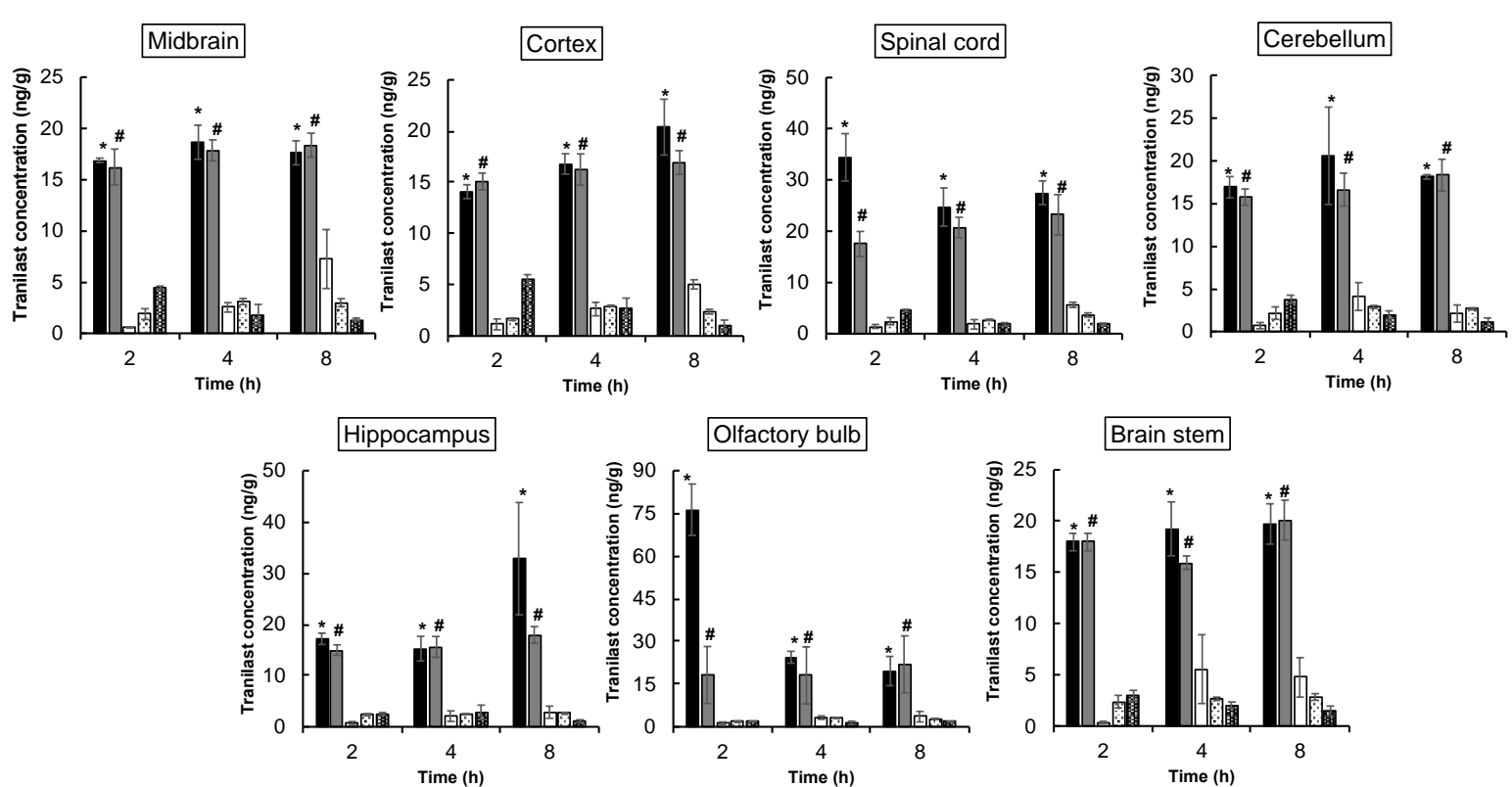

Figure 4. Concentration of tranilast in brain regions. $\mathrm{TL}_{M}(\boldsymbol{\square}), \mathrm{TL}_{\mathrm{ME}}(\boldsymbol{\square}), \mathrm{TL}_{\mathrm{G}}(\square), \mathrm{TL}_{\mathrm{GE}}(\square)$, $\mathrm{TL}_{\mathrm{C}}($ 㘣). Each value represents the mean \pm S.E. $(\mathrm{n}=3-4)$. Significant difference $(p \leq 0.05)$ 640 between "TLM and TLG, TLGE, or TLC; ${ }^{*} \mathrm{TL}_{\mathrm{ME}}$ and $\mathrm{TL}_{\mathrm{G}}, \mathrm{TL}_{\mathrm{GE}}$, or TLC. 

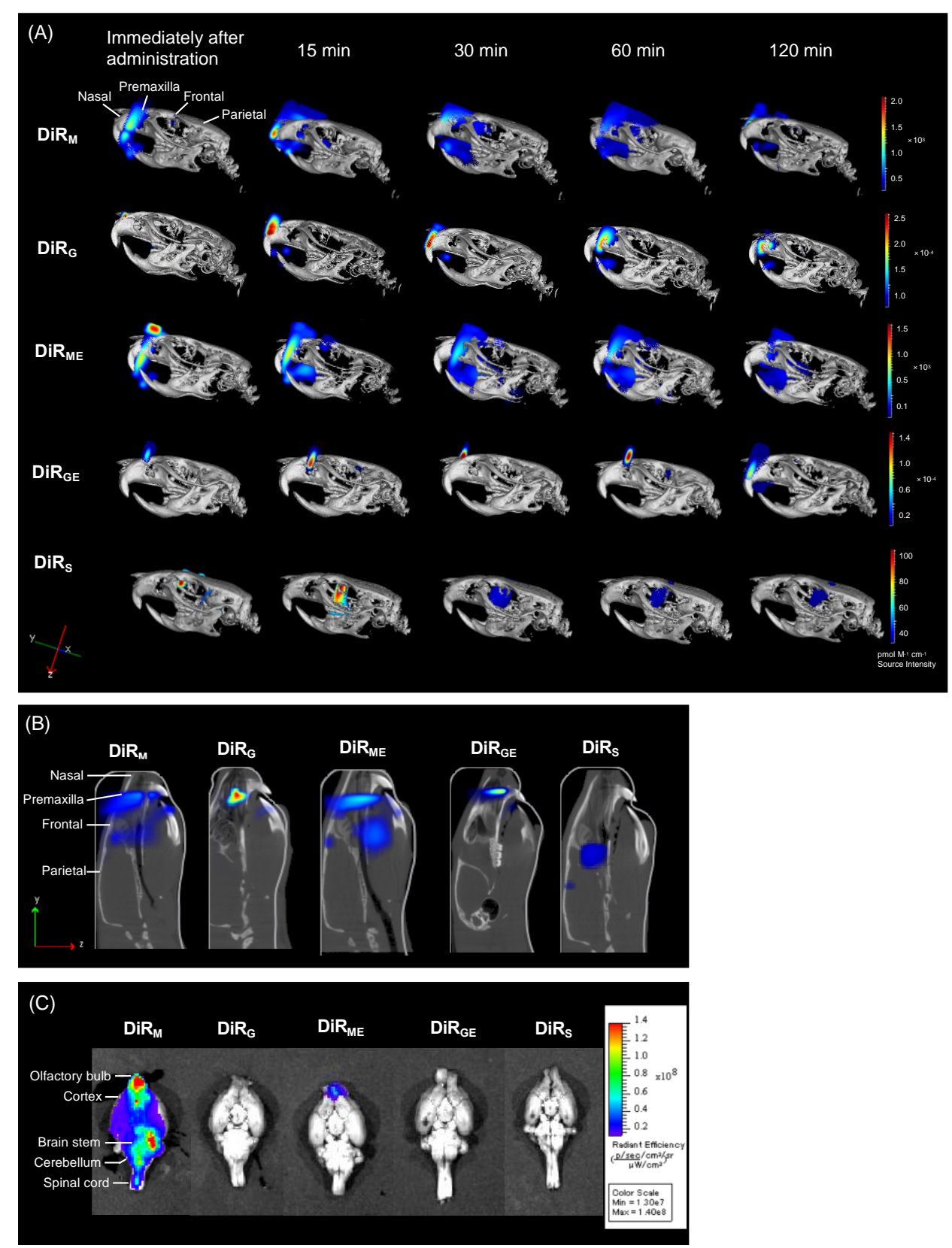

655 Figure 5. Biodistribution of $\mathrm{LC}$ formulations by in vivo fluorescent $/ \mu \mathrm{CT}$ imaging. (A) in vivo fluorescent signals co-registered with X-ray and micro-computed tomography images $(\mu \mathrm{CT})$; (B) Slice images in the lateral direction at $120 \mathrm{~min}$ post-administration; (C) ex vivo fluorescent images of whole brain at 120 min post-administration. Representative images are presented in this figure $(n=3)$. 
TABLES

Table 1. Composition of nasal formulations

\begin{tabular}{l|c|c|c|c|c}
\hline Components (\%) & $\mathrm{TL}_{\mathrm{M}}$ & $\mathrm{TL}_{\mathrm{ME}}$ & $\mathrm{TL}_{\mathrm{G}}$ & $\mathrm{TL}_{\mathrm{GE}}$ & $\mathrm{TL}_{\mathrm{C}}$ \\
\hline Tranilast & 0.5 & 0.5 & 0.5 & 0.5 & 0.5 \\
Monoglycerol ester (MGE) & 20 & 20 & - & - & - \\
Glyceryl monooleate (GMO) & - & - & 20 & 20 & - \\
Pluronic $^{\circledR}$ F-127 & 5 & 5 & 5 & 5 & - \\
Ethanol $_{\text {Purified water }}$ & - & 5 & - & 5 & - \\
PBS (pH 7.4) & 74.5 & 69.5 & 74.5 & 69.5 & - \\
Total & - & - & - & - & 99.5 \\
\hline
\end{tabular}

675

Table 2. Characteristics of LC formulations

\begin{tabular}{cccccc}
\hline Formulations & $\begin{array}{c}\text { Mean particle } \\
\text { size }(\mathrm{nm})\end{array}$ & $\begin{array}{c}\text { Polydispersity } \\
\text { index }\end{array}$ & -Potential $(\mathrm{mV})$ & $\begin{array}{c}\text { Viscosity } \\
(\mathrm{mPa} \cdot \mathrm{s})\end{array}$ & $\begin{array}{c}\text { Entrapment } \\
\text { efficiency }(\%)\end{array}$ \\
\hline $\mathrm{TL}_{\mathrm{M}}$ & $300 \pm 0.65$ & $0.38 \pm 0.28$ & $-24.1 \pm 0.23$ & 334.6 & $90.5 \pm 0.46$ \\
$\mathrm{TL}_{\mathrm{ME}}$ & $256 \pm 0.39$ & $0.40 \pm 0.13$ & $-35.0 \pm 0.19$ & 913.7 & $85.8 \pm 0.52$ \\
$\mathrm{TL}_{\mathrm{G}}$ & $179 \pm 0.53$ & $0.13 \pm 0.93$ & $-15.5 \pm 0.16$ & 1120.5 & $70.6 \pm 0.45$ \\
$\mathrm{TL}_{\mathrm{GE}}$ & $404 \pm 0.46$ & $0.33 \pm 0.88$ & $-16.1 \pm 0.19$ & 2237.2 & $53.8 \pm 1.02$ \\
\hline
\end{tabular}

680 Table 3. Pharmacokinetic profile of tranilast after intranasal administration

\begin{tabular}{|c|c|c|c|c|c|c|c|c|c|c|c|}
\hline & \multicolumn{4}{|c|}{ Plasma } & \multicolumn{4}{|c|}{ Whole brain } & \multirow[b]{2}{*}{${ }^{*} K p$} & \multirow[b]{2}{*}{$\%$ DTE } & \multirow[b]{2}{*}{$\%$ DTP } \\
\hline & $\begin{array}{l}C_{\max } \\
(\mathrm{ng} / \mathrm{mL}) \text { i.n. }\end{array}$ & $\begin{array}{l}A U C_{(0-8)} \\
(\mathrm{ng} h / \mathrm{ml}) \text { i.n. }\end{array}$ & $\begin{array}{l}C_{\max } \\
(\mathrm{ng} / \mathrm{mL}) \text { i.v. }\end{array}$ & $\begin{array}{l}A U C_{(0-8)} \\
\text { (ngh/ml)i.v. }\end{array}$ & $\begin{array}{l}C_{\max } \\
(\mathrm{ng} / \mathrm{mL}) \text { i.n. }\end{array}$ & $\begin{array}{l}A U C_{(0-8)} \\
(\mathrm{ng} \cdot \mathrm{h} / \mathrm{ml}) \text { i.n. }\end{array}$ & $\begin{array}{l}C_{\max } \\
(\mathrm{ng} / \mathrm{mL}) \text { i.v. }\end{array}$ & $\begin{array}{l}A U C_{(0-8)} \\
\text { (ngh/ml)i.v. }\end{array}$ & & & \\
\hline TLM & $33.9 \pm 7.03$ & $71.4 \pm 11.4$ & - & - & $19.4 \pm 1.50$ & $94.5 \pm 3.68$ & - & - & 3.4 & 384 & 73.9 \\
\hline TLME & $346 \pm 48.6$ & $725 \pm 33.1$ & - & - & $19.4 \pm 1.07$ & $114 \pm 5.92$ & - & - & 0.66 & 45.6 & -119 \\
\hline $\mathrm{TL}_{\mathrm{G}}$ & $61.0 \pm 12.0$ & $142 \pm 9.13$ & - & - & $8.84 \pm 3.63$ & $22.7 \pm 6.41$ & - & - & 1.09 & 46.3 & -115 \\
\hline $\mathrm{TL}_{\mathrm{GE}}$ & $10.0 \pm 1.71$ & $56.4 \pm 8.13$ & - & - & $2.75 \pm 0.06$ & $15.5 \pm 2.73$ & - & - & 0.46 & 79.6 & -25.5 \\
\hline TLC & $181 \pm 31.3$ & $189 \pm 16.2$ & $1064.3 \pm 5.23$ & $1018.6 \pm 8.19$ & $3.46 \pm 0.31$ & $9.55 \pm 2.17$ & $116.5 \pm 4.31$ & $351.5 \pm 4.16$ & 0.13 & 14.6 & -582 \\
\hline
\end{tabular}

i.n.: intranasal; i.v.: intravenous; ${ }^{*}$ tissue-to-plasma concentration ratio (whole brain TL concentration/plasma TL concentration) at $8 \mathrm{~h}$. 